\title{
Creative Processes during a Collaborative Drawing Task in Teams of Different Specializations
}

\author{
Olesya Blazhenkova ${ }^{1 *}$, Maria Kozhevnikov² \\ ${ }^{1}$ Sabancı University, Istanbul, Turkey \\ ${ }^{2}$ National University of Singapore, Singapore \\ Email: ^olesya@sabanciuniv.edu,psymaria@nus.edu.sg
}

How to cite this paper: Blazhenkova, O., \& Kozhevnikov, M. (2020). Creative Processes during a Collaborative Drawing Task in Teams of Different Specializations. Creative Education, 11, 1751-1775. https://doi.org/10.4236/ce.2020.119128

Received: August 4, 2020

Accepted: September 20, 2020

Published: September 23, 2020

Copyright $\odot 2020$ by author(s) and Scientific Research Publishing Inc. This work is licensed under the Creative Commons Attribution International License (CC BY 4.0).

http://creativecommons.org/licenses/by/4.0/ (c) (i) Open Access

\begin{abstract}
The present research examined creative drawing processes in teams of gifted adolescents with different educational specializations, including teams with homogeneous (the same specialization) and heterogeneous (mixed specialization) composition. Based on the converging evidence from protocol and Linkography analyses, we identified the differences in frequency and dynamic distribution of distinct creative processes between the different teams specializing in visual art, natural science, humanities, as well as mixed specialization teams. Visualization processes played a crucial role for visual art, science, mixed, but not for humanities teams. All teams except humanities had visual planning earlier in the creative process. Visual artists' visualization processes developed prominently and continuously throughout all stages of creative production with the main focus on visual aesthetics while for scientists, they developed more discreetly, and in conjunction with understanding of function. Mixed and visual art teams shared many similarities, and they had the highest level of integration between the ideas expressed during their creative processes. Mixed team had higher frequency of organizational processes, indicating coordination and organization challenges due to their diversity. The results of this research show the importance of considering differences in visualization profiles while composing teams of different specializations.
\end{abstract}

\section{Keywords}

Creative Processes, Visualization, Teamwork, Linkography, Artistic Creativity, Scientific Creativity 


\section{Creative Processes during a Collaborative Drawing Task in Teams of Different Specializations}

The present research examined creative processes during collaborative drawing task in teams of different specializations. Over the last two decades, professional work in different fields, including artistic and scientific domains, has increasingly involved collaborative practice, while solo authors producing creative works become less frequent (Wuchty, Jones, \& Uzzi, 2007). The growing interest in interdisciplinary collaborations creates new educational and professional demands, and preparedness to work in multidisciplinary teams becomes a required skill expected from both engineering (Hirsch et al., 2001; Terenzini, Cabrera, Colbeck, Parente, \& Bjorklund, 2001) and visual art and design (Stevelt-Kaser, Pennington-Busick, \& Rhoades, 2004) schools. Along with development of new technologies and new visual media, the role of visual communication is constantly growing, bringing together specialists from various creative fields (e.g., visual art, design, computer science, engineering etc.) to work in mono- and multidisciplinary teams and to share visual information. However, understanding of collaborative processes behind interdisciplinary team interaction, and how visual information is processed and shared among the team members with different visualization profiles such as visual artists and scientists has been limited.

Most previous studies on collaborative team performance were conducted in the fields of design research and organisational psychology. Design research has been mostly interested in the description of creative processes in architectural and engineering domains. To discover general patterns emerging in different collaborative design situations, it examined the characteristics of design activities, team interaction processes as well as communication behaviour that supports collaboration during the creative process (Austin, Steele, Macmillan, Kirby, \& Spence, 2001; Dong, 2005; Kvan, 2000; Sonnenwald, 1996). In particular, it has explored how the characteristics of creative processes (e.g., dynamics of idea development and their interconnectedness) can predict the quality of a creative product (e.g., based on experts' estimates). Linkography analysis of interconnectedness between the ideas during design process (Goldschmidt, 1990, 1992; Kan \& Gero, 2008) has been frequently implemented in this research to examine creative processes either in a single designer or in a team of designers. Linkography research reported that the most creative and productive works, highly evaluated by the experts, had denser and more integrated structure of Linkographs (Goldschmidt \& Tatsa, 2005; Van der Lugt, 2003). Moreover, Linkograph metrics were found to reflect the differences between expert and novice designers (Cai, Do, \& Zimring, 2010; Kan, Bilda, \& Gero, 2007).

While design research primarily focused on qualitative descriptions of team creative processes, organizational psychology research focused more on how team composition factors (e.g., heterogeneity vs. homogeneity) and team processes (e.g., implemented strategies and communication) affect team performance (Aggarwal \& Woolley, 2013; Hackman \& Hackman, 2002; Salas, Sims, \& Burke, 
2005; Woolley, Hackman, Jerde, Chabris, Bennett, \& Kosslyn, 2007; Woolley, Chabris, Pentland, Hashmi, \& Malone, 2010). It has been argued that greater similarities between team members' mental representations (shared mental model) lead to better communication, improved anticipation of each other's actions, and better coordination of activities, eventually resulting in more efficient performance (Banks \& Millward, 2000; Cannon-Bowers, Salas, \& Converse, 1993; Klimoski \& Mohamed, 1994; Kraiger \& Wenzel, 1997; Mesmer-Magnus \& DeChurch, 2009). At the same time, dissimilarities between team members might allow to bring unique perspectives to the task and tap a broader array of relevant information (Egan, 2005; Milliken, Bartel, \& Kurtzberg, 2003).

Overall, the majority of the previous studies on team creativity explored creative collaborative processes of either artists, architects, engineers or scientists (Atman, Chimka, Bursic, \& Nachtmann, 1999; Bilda, Costello, \& Amitani, 2006; Dunbar, 1999; Hagaman, 1990; Kan \& Gero, 2005; Stokols, Hall, Taylor, \& Moser, 2008) but did not explicitly compared creative processes in homogeneous and heterogeneous teams composed of individuals with different specializations whose work involve visualization. At the same time, cognitive psychology and neuroscience studies demonstrated qualitative and quantitative differences between visual art, sciences, and humanities professionals in their ways to process visual information (Blazhenkova \& Kozhevnikov, 2010; Kozhevnikov, Kozhevnikov, Chen, \& Blazhenkova, 2013; Kozhevnikov, Kosslyn, \& Shepard, 2005). In particular, research showed the dissociation between object visualization (i.e., processing visual information about the object or scene appearances in terms of their visual properties such as color, shape and texture) and spatial visualization (i.e. processing visual information about spatial relations, performing mental spatial transformations and manipulations). It was reported that visual artists excel on object imagery tasks, while scientists and engineers excel on the tasks that require spatial visualization. Furthermore, the dissociation between object and spatial visualization has been related to artistic and scientific creativity, respectively, and these two types of creativity appeared to be significantly different in terms of visualization processes they involve (Kozhevnikov et al., 2013). Recent research (Blazhenkova \& Kozhevnikov, 2016) has shown that the drawings created by teams of different visualization profiles were different in their visual object vs. spatial characteristics as well as they were evaluated differently by the experts from different professional domains. Visual art and mixed teams' drawings were evaluated as the highest in artistic quality, science teams were evaluated as the highest in concept clarity, whereas humanities drawings were evaluated as the lowest on both criteria.

The present research is a part of a larger study (Blazhenkova \& Kozhevnikov, 2016). This previous study showed that different specialization teams, composed of individuals with diverse visualization profiles, produced qualitatively different creative outputs. The drawings produced by these teams were found to be different in terms of their visual characteristics as well as they were evaluated differently in terms of artistic quality and conceptual clarity by the experts from 
different professional domains. While the focus of that study was on the analyses of team's drawn outputs in terms of their visual characteristics, the main goal of the present research was to explore creative processes, with a specific focus on visualization, in teams composed of individuals with different visualization profiles (specializing in visual arts, sciences, or humanities), including groups with homogeneous (the same specialization) and heterogeneous (mixed specialization) composition. In particular, we were interested in the differences between these teams' communication processes, level of integration between the ideas expressed during the creative processes as well as the process of idea development. Based on previous research findings that creativity of individuals with different visual profiles are different (Kozhevnikov et al., 2013), we expected creative processes to be considerably different in teams of different specializations (visual artists, scientists, humanities). We also expected creative processes of heterogeneous groups to be different from those of homogeneous groups.

The first goal was to investigate the role of visualization in creative processes of teams specializing in visual art, sciences, and humanities as well as mixed specialization team. The second goal was to explore the differences between the teams' visual and non-visual collaborative processes throughout the duration of the entire creative activity. In the current study, we implemented protocol analysis of participants' communications during their collaborative creative work. The protocol analysis categories were examined and compared between the teams in terms of their frequency distribution as well as dynamic frequency distribution throughout the duration of the creative process. Furthermore, to examine the teams' differences in the level of integration between the ideas expressed during the creative process, we used the Linkography method (Goldschmidt, 1990). Finally, we explored the relationship between the characteristics of team processes (i.e., Linkography metrics tapping different parameters of idea development examined in the current study) and team performance characteristics (i.e., expert' estimates of the drawings, revealed in Blazhenkova \& Kozhevnikov, 2016, study).

\section{Method}

The current study is a part of a larger study (Blazhenkova \& Kozhevnikov, 2016) which implemented a collaborative open-ended creative task (drawing an "unknown planet") and used adolescent sample (students from schools for gifted children, specializing in visual arts, science, humanities or multiple disciplines). Using schoolchildren allowed to examine creative drawing in members of different specializations and to present them a comparable level of challenge to the teams with different specializations in ecologically valid settings school settings (otherwise, such a creative drawing task may be not ecologically valid for adult professionals) Previous research showed that adolescents' visualization abilities are nearly developed (Blazhenkova, Kozhevnikov, \& Becker, 2011), and their object or spatial visualization abilities relate to their aptitudes in visual art or sciences specializations (Kozhevnikov, Blazhenkova, \& Becker, 2010). 


\subsection{Participants}

The participants were recruited from Russian specialized schools for gifted children, which use advanced curricula, concentrating on a specific discipline (visual art, science, or humanities), and involving university-level faculty as teachers. Overall, 28 adolescents (16 females; 11 - 17 years old; $M$ age 14.60) formed four specialization teams (with 6 - 8 participants in each team): "visual art", "science" and "humanities" homogeneous teams (from schools for gifted with the above specializations) as well as "mixed" heterogeneous team (from a school that admits gifted students from a variety of domains and offers an advanced multidisciplinary curricula).

\subsection{Procedure and Materials}

The study was conducted in the students' typical school environment such as classrooms. Each team received a large piece of paper (A1 size) placed on a table and various drawing materials (e.g., pencils, crayons, pastels, and gouache). Participants were instructed to imagine and to draw an unknown planet as a team. The current creative task allowed a variety of approaches encouraging the use of representations from different domains (e.g., science, visual art, and humanities) and divergent outcomes. Participants could move freely around the table while drawing and use any materials and any space on the paper. Students were encouraged to share and communicate with each other. The maximum time allotted to the task was 45 minutes (a typical time of a school lesion), and they could finish their work earlier upon agreement. After completion of the drawings, participants were briefly interviewed about their experiences. Drawing processes and the subsequent interviews were video recorded and transcribed.

\section{Results}

\subsection{Protocol Analysis of Collaborative Processes}

The main goal of the current protocol analysis was to examine and compare collaborative processes in teams of different specializations. First, a coding scheme that distinguished between different categories of collaborative processes was developed. Second, the frequency of the identified categories was examined. Third, the dynamic distribution (frequency in time) of different categories was analyzed across the duration of teams' creative processes.

Coding scheme development. The coding scheme was developed based on an examination of video recordings from all the teams. The coding scheme was modified from Kan \& Gero's (2009) creative design model to include more elaborated categorization of visualization processes and other aspects of collaborative activities during the drawing task. While developing the present coding scheme, we paid attention to visualization processes as well as to participants' discussions related to drawing activity. First, the three main stages of creative processes were identified: Planning (discussions about future drawing and considering different possibilities before their execution), Drawing (implementing 
the ideas in the actual drawing), and Evaluation (interpreting and evaluating the drawings). In our protocol analysis, we further identified different categories representing specific processes occurring during each stage, as described below.

Planning Stage. During this stage, participants discussed their future drawings in terms of visual appearances. They mentally simulated different possible visual outcomes as well as planned how their future planet would function. Thus, the main three categories of teams' activities during Planning Stage were: Planning of Visual Appearances, Mental Simulations, and Discussion of Functions.

The Planning of Visual Appearances category included suggestions regarding the planets' appearances, e.g., in terms of color, shape, size, detail, specific visual properties, or object presence ["Make it like Saturn, but with two rings"; "Let's draw things on the planet in an enlarged size, with some particular sights"]. The Mental Simulations category included visualizations of possible outcomes of manipulating visual appearances. For example, it included imagining the results of mixing colors ["If we mix these colors it will be a mess"], changing visual properties [in response to a suggestion to help in drawing cracks: "If I draw this, then it would be a different style and width"], altering composition and locations ["If we place a cube here, then we could turn it like this, and could draw here as well, from this side ... and in different directions ...-Look how cool-if this is water here, then here would be its cross-section"]. The Discussion of Functions category included discussions of planet functioning, in terms of the nature or physics governing the planet ["It can be a liquid planet with very strong attractive forces"; "This planet will have off-centre gravity"] as well as descriptions of living forms and possibilities of life ["No one can survive on this planet"; "Lizards and the penguins and whales inhabit the planet"].

Drawing Stage. Drawing stages were often accompanied by different organizational processes controlling participants' collaborative activities and guiding the flow of the drawing process, as well as by discussions of restrictions and setting constraints on the content of the drawing in terms of its aesthetic appearance or functional value. Thus, two main categories were identified during drawing stages: Organizational Processes and Setting Restrictions categories.

The Organizational Processes category included communications regulating the organization of work process, e.g., task delegation, start/stop commands, distributing roles, votes, encouragements, and control or fairness ["We should start drawing now"; "Wait to draw-we need to discuss first"; "-Who is generally agreed that we draw only landscape? (voting with raising hands) - Yeah ...”].

The Setting Restrictions category included discussions of different constraints on the content of the drawings and on the drawing process itself: functional restrictions, related to the violation of physics laws and logical rules ["This is too big for a tree ...-If here are couple thousands of kilometres, then how is it possible to be a tree?"]; aesthetic requirements, related to the violation of the laws of aesthetics) ["Blue splashes on the blue sky will be invisible"; Light should go 
from this angle, so here it will be darker; No! Don't use violet, it's too bright for this]; material/technical constraints (e.g., limited given drawing materials, allotted space etc.); instructional restrictions (rules and restraints that participants set for themselves based on their interpretation of the task instruction, e.g., what can be considered as unknown planet, the given time limit to draw the planet); and restrictions related to difficulties drawing a proposed object (e.g., due to lack of drawing skills).

Evaluation stage. Evaluation stages included only one category-Product Evaluations, which comprised of interpretations and evaluations of already drawn content in terms of their visual characteristics and interpreted conceptual meanings of the drawn ["-Are you sure that these are apples?-Maybe not-Maybe these are berries ..."]. All other discussions irrelevant to the creative processes were assigned to an Irrelevant (IR) category which included discussing personal issues, school activities, etc. Since Planning of Visual Appearances, Mental Simulations and Product Evaluations categories consisted of discussions related to visual appearances and visual characteristics of the drawings, we grouped them into a Visualization Processes combined category for further analysis. It should be noted that these three categories emphasized different aspects of visualization. Planning of Visual Appearances was formulated declaratively, implying the execution of the proposed idea. Mental Simulations were formulated as questions, which required performing visualization of possible outcomes and inspecting how the future drawing would look like in case of certain drawing actions. Product Evaluations also required visualization and image interpretation; however, the inferences were made from already drawn content, based on external visual representations.

Frequency distribution of protocol analysis categories. Two judges segmented the transcripts into separate meaningful utterances, usually a length of a sentence ["It is not clear why the atmosphere is green" or "Let's draw a ring around the planet"]. Each segment represented a separate act of reasoning or a coherent proposition related to what is being drawn. Overall, 442 segments were identified in the visual art team transcript, 341 segments in the science team transcript, 310 segments in the humanities team transcript, and 388 segments in the mixed team transcript. Then, each segment was assigned to one of the categories described in the coding scheme development section by two independent judges. The agreement between judges was $86 \%$. All the discrepancies in classifying the segments into the categories were resolved by consensus. The frequencies of each category across different teams are presented in Figure 1.

For the visual art team, the most distinct and prevalent category was Planning of Visual Appearances (47.7\%), so that about a half of the visual artists' discussions were devoted to visual properties of the planet and its appearance, e.g., in terms of color and detail of the proposed objects. The next frequent category was Mental Simulations (16.1\%), followed by Setting Restrictions (12.9\%), and Product Evaluations (12.3\%). It is interesting that Setting Restrictions was mostly related to visual appearances and aesthetic requirements (e.g., color blending 


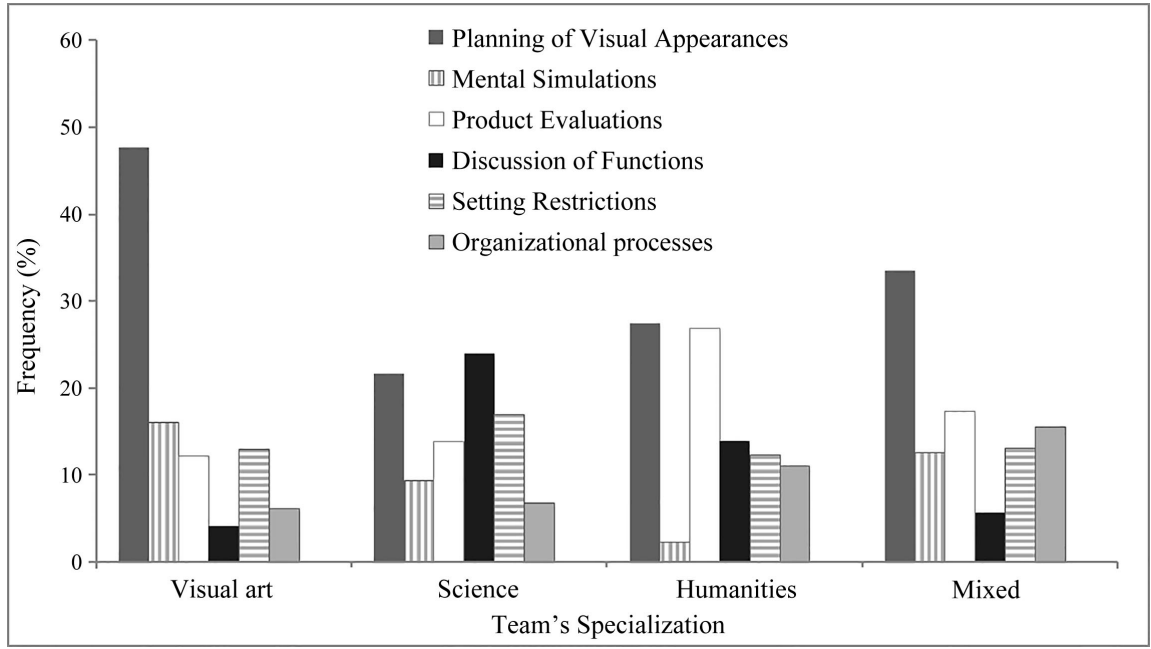

Figure 1. The frequencies of each category of protocol analysis.

rules, light source, rules of rendering 3D objects, use of the golden ratio etc.). Overall, $76 \%$ of all the discussions were devoted to the three categories related to Visualization Processes. Visual artists had very little Discussions of Functions (4.1\%).

In the science team, in contrast to visual artists, the most distinct and prevalent category was Discussion of Functions (24\%). Most of the scientists' Discussion of Functions (80.5\% within this category) were related to their planets' physical nature, and they often referred to knowledge about physical laws and astronomy (e.g., how the planet would spin, its gravity, chemical makeup, magmatic activity, presence of rings etc.), or devoted to descriptions of living forms and possibilities of life. Interestingly, Planning of Visual Appearances constituted only $(21.7 \%)$ of all the discussions, while in all other teams Planning of Visual Appearances was the most frequent category. Notably, Planning of Visual Appearances in the science team were also often related to functional properties ["So, the atmosphere will be dark-green because it is made from chlorine"; "Let's make it blue, it will be a blue giant"]. Setting Restrictions (12.9\%) discussion were almost 9 times more often related to functional rather than aesthetic restrictions.

In the humanities team, about half (56.5\%) of all the discussions belonged to the two categories related to Visualization Processes such as Planning of Visual Appearances (27.4\%) or to Product Evaluations (26.8\%). Interestingly, the humanities team was the only team that almost did not have MS (2.3\%). During Planning of Visual Appearances, the humanities team did not explicitly discuss either pictorial details or spatial configuration, but rather simply named different objects to be drawn. The highest number of Product Evaluations in the humanities team in comparisons with all other teams resulted from their attempts to interpret the meaning of what they had drawn only after it was drawn (and in some cases, they were unable to unambiguously identify the drawn object). The less frequent categories in the humanities team were Discussion of Functions 
(13.9\%), Setting Restrictions category (12.3\%), and Organizational Processes (11\%).

The profile of the mixed team was similar to the visual artists' profile which was somewhat surprising, given that there was only 1 visual artist and 5 scientists in this team. Overall, more than half (63.4\%) of their discussions were devoted to Visualization Processes. Planning of Visual Appearances was the most distinct and frequent category (33.5\%), while Discussion of Functions was the least frequent $(5.7 \%)$. In comparison to the other teams, they had relatively large number of discussions on Organizational Processes (15.5\%), possibly due to the need for an extra effort in the coordination of different individuals' views throughout the drawing process. As for Setting Restrictions (13.1\%), mixed teams had the most frequent discussions of instructional restrictions, which indicated that they regularly attempted to reinterpret the task instructions and to follow them. Their discussions of aesthetic requirements were only about 1.5 times more frequent than discussion of restrictions related to functions, which suggests that, in contrast to visual artists, the mixed team was both concerned about aesthetic and functional restrictions to a comparable level.

Dynamic distribution of all the categories defined in protocol analysis. Figure 2 represents the dynamic distribution of all the defined categories during the whole period of creative process.

Planning of Visual Appearances in visual art, science, and mixed teams were more frequent in the beginning, and then dropped off towards the end of the drawing process. In contrast, the humanities team did not follow this trend, and there was a considerable proportion of Planning of Visual Appearances at the end of the process. PE in visual art, science, and mixed teams appeared later in the process of drawing and became more frequent towards the end of their drawing. In contrast, the humanities team had the opposite trend: their evaluations started at the very beginning of the process and dropped at the end. Mental Simulations had various dynamic frequency fluctuations in different teams; however, in the visual art, mixed team, and science teams, they were more or less present throughout the whole drawing process, while in humanities they were only present in several rare occurrences. Discussion of Functions in the visual art, mixed and humanities teams, appeared mostly in the middle and at end of their creative process. In contrast, the science team started to discuss functions along with Planning of Visual Appearances from the very beginning (the dynamic distribution of these two categories was quite similar), and finally dropped at the end. In the science team, Discussion of Functions either preceded or developed along with Planning of Visual Appearances, suggesting that their visualization and planning of functions were closely related processes. Possibly, Planning of Visual Appearances was constructed based on their understanding of planet functioning. $S R$ and OP in visual art and mixed teams steadily continued throughout the process, with a gradual decline towards the end. However, in science and humanities teams, restrictions appeared in more pronounced and 

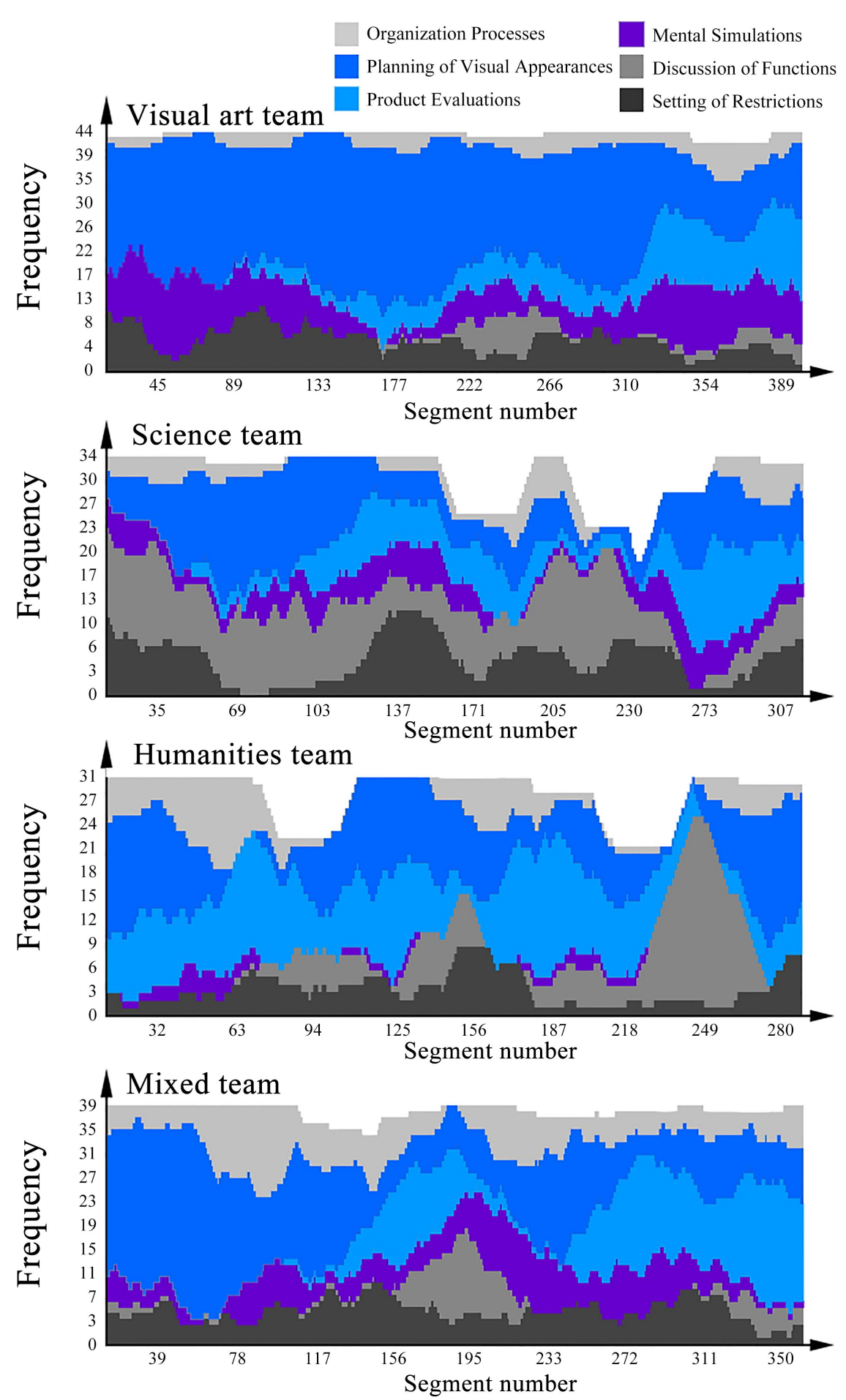

Figure 2. Dynamic distributions of all categories of protocol analysis. Note: All Visualization Processes are represented in the shades of blue, and Non-Visual Processes are represented in the shades of gray.

discrete peaks. Overall, Visualization Processes were continuously prevalent over non-visual processes and were relatively equally distributed throughout the process of drawing for the team of visual artists as well as for the mixed team, 
but less equally distributed for the science and humanities teams.

Time spent for planning activities. While analyzing video recordings, we noticed the difference between teams in time devoted to planning activities, that is, the time from getting instructions to starting drawing (i.e., discussions and planning of future drawings or drafting some preliminary sketches of planets' appearances), and the time that they spent for drawing itself. Humanities team had almost no preliminary activities ( $6 \%$ of the total time) and started to draw right after getting the instructions, in contrast to all other teams that spent considerable amount of time for preliminary activities (visual art team- $51 \%$, science team $-30 \%$, and mixed team $-27 \%$ of the total time). Notably, during these preliminary planning activities, humanities team did not do any drafting and sketching while visual planning was a typical for visual artists, scientists and mixed teams.

Discussion. The results of protocol analyses revealed that all teams differed in their categories' frequency profiles and their dynamic distribution, although there were some similarities between specific teams. The team of humanities had a distinct frequency profile and dynamic distribution of categories in comparisons with other teams. In particular, the humanities team had remarkably low frequency of Mental Simulations, but it had the highest frequency of Product Evaluations category, suggesting that the humanities team used visual imagery primarily for analysis. Furthermore, the humanities team had the opposite pattern of dynamic distribution across all Visualization Processes categories from that of visual artists, scientists, or mixed teams. In contrast to all other teams, the humanities team did not have Planning of Visual Appearances early in the process, but tended to have more planning towards the end, and they had more Product Evaluations at the very beginning and less towards the end of their creative process. These results suggest that imagery of the humanities team might have a different function than imagery used by other teams. Specifically, the humanities team might not use visualization to create or predict new knowledge, but rather for interpretation of already created visual representations. In contrast, the teams of visual artists, scientists, as well as the mixed team were planning visual appearances early in the process, which preceded drawing, and tended to evaluate and interpret the drawings after their completion. This indicates an important functional role of visualization in creative processes of these teams, consistent with Blazhenkova and Kozhevnikov's (2010) findings that visual artists and scientists used visual imagery at the very early stages of their work to guide and inspire their further creative process.

Despite the above similarities, there were also marked differences in the categories' frequency profiles and their dynamic distribution between the team of scientists and the visual art and mixed teams. In visual art and mixed teams, Planning of Visual Appearances was the most frequent category, while the most prevalent category for scientists was Discussion of Functions, which either preceded or developed along with other Visualization Processes. Thus, scientists were especially concerned with the functions of their drawn objects, consistent 
with previous research (Blazhenkova \& Kozhevnikov, 2010; Gooding, 2004) that the main function of visual imagery for scientists is to facilitate their understanding of functionality of the system (e.g., outline its structures, schematize the parts of a system and their interactions, and understand relationships among its parts). Furthermore, while visual art as well as mixed teams had more smoothly and continuously distributed Visualization Processes suggesting the continuous role of visualizations at different stages of creative process, the team of scientists exhibited more discrete and abrupt development of Visualization Processes, suggesting that they use visualization only during specific periods of the creative process. Previous research (Blazhenkova \& Kozhevnikov, 2010) reported that professional scientists use visual imagery primarily during specific stages of their work (e.g., planning, problem solving), while visual artists use visual imagery continuously during all stages of their art-making creative work (e.g., as inspiration for beginning artwork, during the planning, execution, and estimating the artwork).

The mixed team shared many similarities with visual artists, both in terms of frequency of different protocol categories and their dynamic distribution. Like visual artists and unlike scientists, they had a high frequency of Planning of Visual Appearances and a low frequency of Discussion of Functions. The mixed team had the highest frequency of Organizational Processes in comparison to other teams, probably because they made an extra effort in coordination of their different individuals' views throughout their creative process.

\subsection{Linkography Analysis}

In order to examine the teams' differences in the level of integration of their ideas expressed during the creative process and idea development, we used Linkography analysis method (Goldschmidt, 1990, 1995; Kan et al., 2007). This method is widely used in research field that studies creative processes in design (e.g., Goldschmidt, 1990, 1995; Kan et al., 2007). It aims to reveal the conceptual interconnectedness between the ideas, i.e., a meaningful relationship between the ideas reflected in different protocol segments across the whole length of the creative process. In the Linkography analysis, same as in the above protocol analysis, the transcripts are decomposed into segments (also referred as "moves") and then all the conceptually related segments were linked. The links are established based on determining meaningfully associated ideas that occurred in the time sequence of the protocol and then by connecting these related ideas, identified in one or more segments, to each other. A graphical representation which displays the structure of a creative process by tracing the associations of every idea, called a Linkograph, is constructed to represent the patterns of associations between the ideas proposed during the creative process. Backlinks are links that connect to previous segments and indicate the paths that had led to idea generation, while forelinks connect to subsequent segments and bear evidence about contribution to the production of further ideas (see an example of a fragment of a Linkograph in Appendix A). 
There are several common structures identified in a Linkograph such as chunks (i.e., a group of segments that are almost exclusively linked among themselves), webs (i.e., a large number of links among a relatively small number of segments), and sawtooth tracks (i.e., successive segments, each of which is linked only to the preceding and following segments). Sawthooth tracks characterize the sequential, stepwise development of ideas without referring to earlier ideas, while chunks and webs characterize meaningful clusters of ideas that were developed in connection with each other. Goldschmidt (1992) reported that Linkographs of the most creative and productive work (those that were highly evaluated by the judges) had more chunks and more webs.

Link Index indicates the degree of the "completeness" or the level of integration between the ideas in a creative process. It is computed as the ratio between the number of links and the number of segments, thus representing the density of a Linkograph (Goldschmidt, 1995). Critical Moves are segments which have the greatest number of links in the Linkograph, representing important or key strategic decision points that appeared during the creative process. High values of Link Index and Critical Moves were found to be related to higher estimates of creative products by experts (Goldschmidt \& Tatsa, 2005; Van der Lugt, 2003). Entropy measure was introduced by Kan and Gero (2005) as a measure of link integration, based on Shannon's (1948) information theory. In this theory, the amount of information carried by a message is based on the probability of its outcome. The assumption is that the least predictable (or most random) sequence of events should carry the maximum information. The assumption behind the computation of Entropy is that a randomly linked Linkograph (neither poorly linked nor fully saturated) represents a balanced process that holds both integration and diversification of ideas. Based on their findings that expert designers had higher entropy than novice designers, Kan et al. (2007) proposed that Entropy represents the opportunities for idea generation. Entropy correlates with Link Index only until it reaches its maximum, and after its peak, Entropy decreases as more links are formed, implying that in the case of a fully linked linkography, there are no possibilities for the development of new ideas and less opportunity for creative outcomes.

Two types of Entropy could be distinguished: Backlink Entropy and Forelink Entropy, which are measured separately in rows of forelinks and backlinks. Forelink Entropy conceptually reflects the idea generation opportunities in terms of new initiations that lead to the subsequent ideas, while Backlink Entropy reflects the opportunities in building upon previous ideas (Kan \& Gero, 2005). Thus, if an idea is completely novel, it will not have backlinks, and if an idea is weak and do not provide any basis for further idea development, it will have no forelinks. Based on the shape and structure of the Linkograph, researchers can make inferences about the level of integration between the ideas and patterns in their development (see examples of possible Linkograph structures and their interpretations in terms of Entropy in Appendix B).

For the current analysis, all the segments identified in the previous protocol 
analysis were assigned sequential numbers. Then, two independent judges identified all the conceptually related segments. The agreement between the judges was $92 \%$, and all the discrepancies were resolved through consensus. Then, using LiNKODER software (Pourmohamadi \& Gero, 2011) we constructed a Linkograph for each team. Furthermore, we computed Link Index and Entropy measures (Table 1). We also identified Critical Moves as those segments that had both high (exceeding one SD from the mean number of links per segment for the current team) number of backlinks and forelinks. Additionally, we examined to which categories identified in the previous protocol analysis, the critical moves were related as well as the function of Entropy versus time across the whole length of the creative process. Using the LiNKCODER software, we created the graphs for dynamic forelink and backlink Entropy along the sequence of segments (Figure 3).

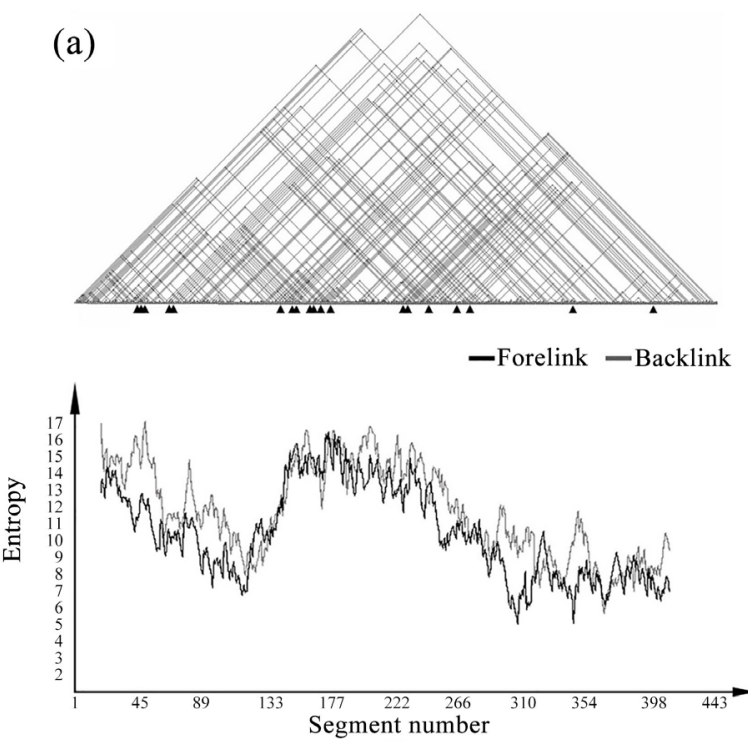

(c)

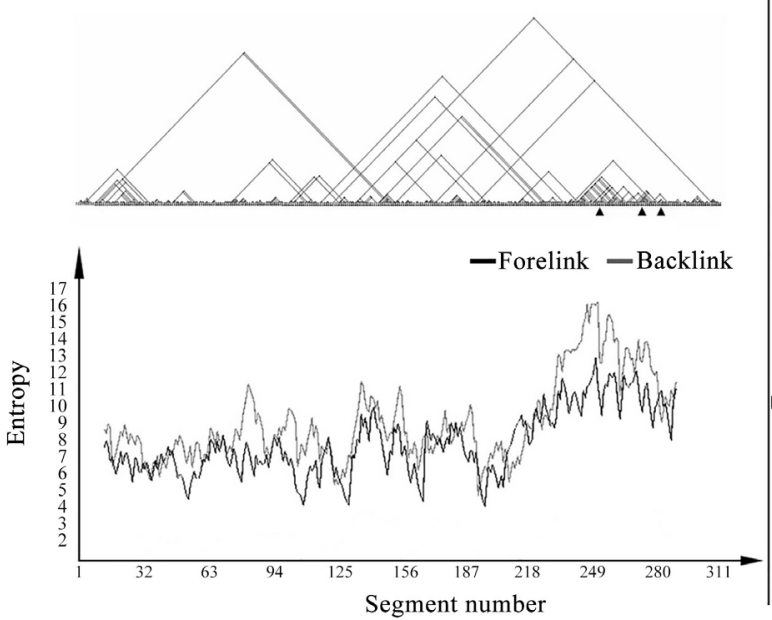

(b)

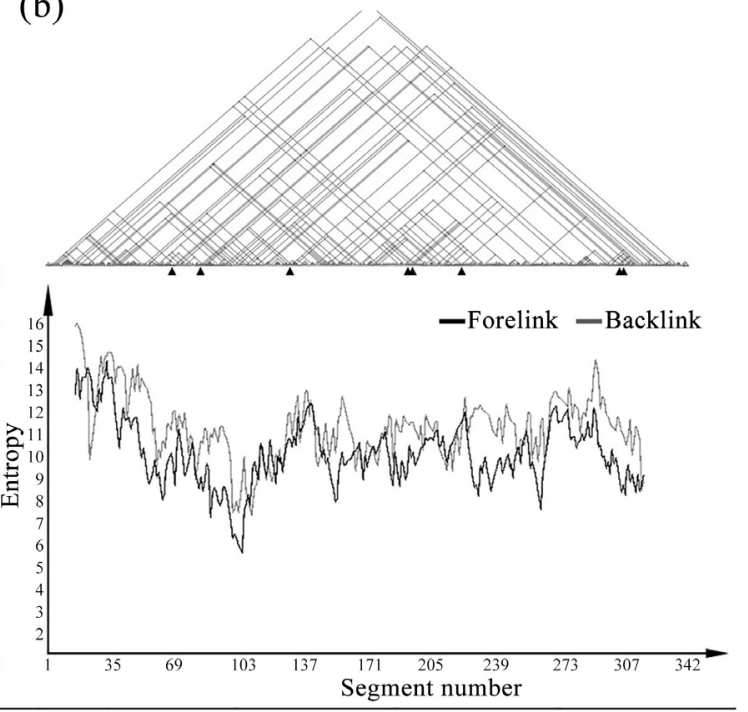

(d)

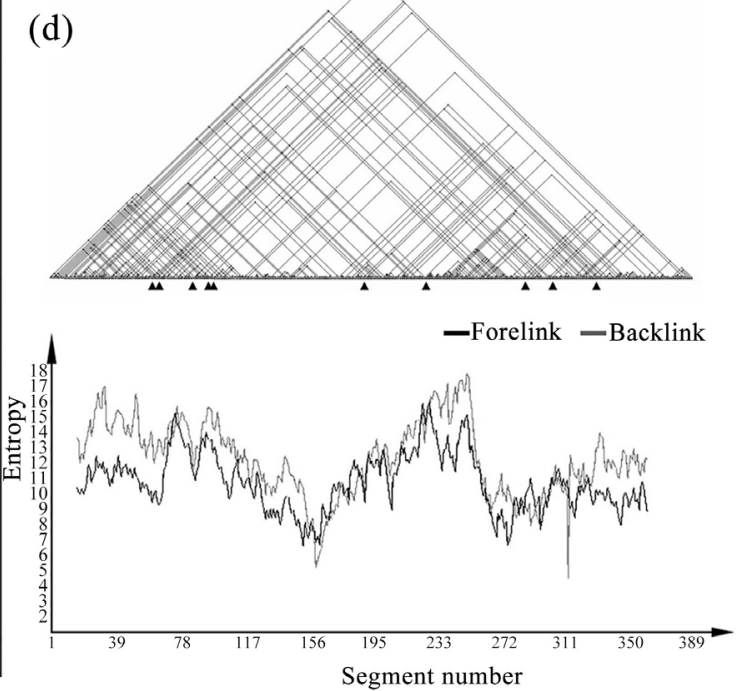

Figure 3. Linkographs and Entropy dynamics of (a) visual art, (b) science, (c) humanities, (d) mixed teams. Filled triangles indicate Critical Moves. 
Table 1. Linkographs statistics for all teams.

\begin{tabular}{ccccc}
\hline & Visual art & Science & Humanities & Mixed \\
\hline Total link number & 806 & 496 & 336 & 753 \\
Link Index & 1.83 & 1.46 & 1.08 & 1.95 \\
Forelink Entropy & 31.42 & 32.09 & 29.86 & 36.55 \\
Backlink Entropy & 47.03 & 39.81 & 25.98 & 45.06 \\
\hline
\end{tabular}

Visual art team. The visual art team had many connections between the Linkograph segments (Figure 3(a)). Compared to other teams, they had a high value of Link Index indicating a high level of integration between the ideas proposed during the process of drawing. Large clusters, chunks, and webs indicate that visual artists' ideas have developed in meaningful complexes with high interconnectedness between each other. There were widespread clusters that linked the ideas proposed at the very beginning of the creative process with the ideas proposed at the end, indicating that visual artists retained an awareness of their initial thoughts and developed them throughout the entire process. There were 19 Critical Moves (13-Visualization Processes, 1-DF). Critical Moves tended to appear more in the beginning and in the middle of the Linkograph, suggesting that many critical decisions were made early in the creative process. Artists had high values of both mean Forelink and Backlink Entropy measures indicating a high potential for idea development. Furthermore, the investigation of Dynamic Entropies revealed the two peaks in the beginning and in the middle of the process that corresponded to the beginning of sketching and actual drawing respectively, and then decreased towards the end. According to Kan and Gero (2005), this suggests that visual artists created many opportunities for idea development at the beginning of drafting, and then these opportunities gradually whittled down.

Science team. The science team had a fairly connected structure of the Linkograph (Figure 3(b)). Scientists, compared to other teams, had an intermediate Link Index, indicating an intermediate level of link interconnectedness. Scientists' Linkograph consisted of webs and sawtooth tracks, which indicates that they had both clustered as well as the successive development of ideas. Similar to visual artists, they also had widespread clusters that linked the ideas proposed at the very beginning of the creative process with the ideas proposed at the very end.

There were 8 Critical Moves (5-Visualization Processes, 3-DF). The Critical Moves were relatively equally distributed in the process. Scientists, as compared to other teams, had relatively high Entropy. Interestingly, scientists, as compared to visual artists, had somewhat higher Forelink Entropy, and lower Backlink Entropy. This may indicate that scientists tended to have more idea generation opportunities in terms of new initiations, unlike visual artists who tended to create more opportunities based on the previous ideas. The Dynamic Entropy graphs demonstrate that Backlink and Forelink Entropies tended to peak in the begin- 
ning of the process, to drop off when drawing commenced, and then to stabilize at an average level. Such dynamics of Entropy indicate that scientists, similar to visual artists, had created the opportunities for idea development in the beginning of the process, but unlike artists, they still had the opportunities at the end of the process.

Humanities team. The humanities team had a poorly connected structure as compared to other teams, most densely connected towards the end of the process (Figure 3(c)). The humanities team, among other teams, had the lowest Link Index which indicates a low level of integration between their ideas. Their Linkograph primarily consisted of successive sawtooth tracks, with only a few webs and chunks, reflecting the predominance of sequential, stepwise development of ideas. There were no widespread clusters that persisted throughout the whole creative process and there were only a few single long links, indicating that the humanities team's ideas were rather transient. Only 3 Critical Moves were identified (1-Visualization Processes, 1-DF, 1-OP). All of them were positioned towards the end of the Linkograph, when the actual drawing was almost completed.

The humanities team had the lowest Entropy among other teams. Unlike other teams, the humanities team had Backlink Entropy higher than Forelink Entropy. The Dynamic Entropy graph demonstrated that Entropy was relatively low at the beginning of the process and tended to peak at the end of the process in contrast to all other teams. This reflects that humanities team discovered their opportunities only towards the end of the creative process.

Mixed team. The structural patterns of the mixed team were very similar to those of visual artists (Figure 3(d)). They had a highly connected structure, most densely connected in the beginning of the creative process. The mixed team also had a high value of Link Index. Similar to the artists, their Linkograph had a clustered structure consisting of chunks and webs, as well as sawtooth tracks. A mixed team also had two noticeable large clusters with elaborated connections; one appeared during the initial planning, and the second during the planning preceding the drawing of the substantial elements of the picture. There were also many long link connections and clusters that ran throughout the whole process. There were 10 Critical Moves (8-Visualization Processes, 2-OP). Overall, their Critical Moves tended to appear both in the beginning and at the end of the process.

Furthermore, the mixed team, similar to visual artists, had high values of both Backlink Entropy and Forelink Entropy measures. The examination of Entropy dynamics revealed two main peaks. Dynamic Entropies revealed the two peaks, one in the beginning and one in the middle of the process, which corresponded to the beginning of sketching and actual drawing, respectively, and then decreased towards the end. This reflects that the mixed team, similar to the visual artists, created the most opportunities for idea development at the beginning and middle of the process.

Discussion. The examination of the Linkographs' structure revealed the dif- 
ferences between the teams in the development and level of integration of their ideas. Visual and mixed teams had the highest level of interconnectedness and integration between their ideas (as reflected in Link Index and Entropy measures). According to the literature (Cai et al., 2010; Goldschmidt, 1990; Kan \& Gero, 2008; Van der Lugt, 2003), Linkographs of the most creative and productive work (those that were highly evaluated by the experts) have higher link interconnectedness. Indeed, in our study, Link of Index and Entropy measures as well as number of chunks and webs were consistent with professional estimates, which rated artists and mixed teams as the highest on Artistic Quality (Blazhenkova \& Kozhevnikov, 2016). However, these Linkograph measures were not related to the experts' estimates of Conceptual Clarity, indicating that not all aspects of creative productivity can be tapped by Linkograph measures of link interconnectedness.

As suggested by Kan and Gero (2007), a highly integrated Linkograph may reflect more holistic processing, while a more discretely clustered structure may represent a sequential process of trying different possibilities and developing ideas one after another. This could explain the results of our study. As was evident from the analysis of videorecordings, visual artists and mixed teams, who had the most highly integrated Linkographs, used a more holistic approach to drawing; started from a global scene and then filled in the details while drawing several objects concurrently. Scientists and humanities, in contrast, drew in discrete units, rendering one thing at a time, and they sequentially proceeded from one element of the drawing to the next one. The humanities team's drawing, although sequential, was less coordinated than that of the scientists, so that many elements of the drawing were not related to each other. Our findings are consistent with Kozhevnikov et al. (2005) reporting the differences between artists and scientists' holistic versus sequential visual processing and relating these differences to different types of visualization.

The examination of Linkograph Dynamic Entropy further revealed the differences in the idea development between the teams of different specializations. Both visual art and mixed teams had two Entropy peaks, one at the very beginning of the process, during planning and sketching phases, and the second one before actual drawing. We suggest that such a pattern occurs because the participants create more opportunities at the beginning of the process, but while approaching the end, they tend to converge on a particular approach (see also Kan \& Gero, 2007). Similar to visual artists and mixed teams, scientists had a high level of Dynamic Entropy at the beginning of the process, however, they did not have the second peak during the drawing, suggesting that the highest level of their idea development happened during the initial planning stage (prior to drawing), and they later created their drawing mainly from these originally generated ideas. Unlike the artists and mixed team, they did not have a decrease in Entropy towards the end; it remained rather stable, indicating that they kept generating ideas and developing new opportunities at some level until the end of 
the process. The Dynamic Entropy of the humanities team increased only at the end, indicating that the humanities team discovered their opportunities and made critical decisions based on the analysis of already drawn pieces, whereas in the beginning they were unable to generate new opportunities based on visualization alone.

\section{General Discussion}

The current research examined creative collaborative processes in teams of different specializations during a creative drawing task. Based on converging evidence from the protocol and Linkography analyses, we identified considerable differences in visualization and non-visual processes between the teams specializing in teams of different specializations.

The results suggest that, overall, visualization plays a significant role for creative processes of visual art, science, mixed teams, but not humanities teams. It was evident from the high frequency of visualization categories in the protocol analysis, from the majority of critical moves of these groups related to visualization, as well as from the frequency of visualization processes involved in planning and prediction. Previous research also supports the crucial importance of visualization in creative work of visual artists and scientists (Blazhenkova \& Kozhevnikov, 2010, 2016; Kozbelt, 2001; Miller, 1996).

Furthermore, the analysis revealed differences between the teams in their use of visualization. For visual artists, visualization processes developed prominently and continuously throughout all stages of creative production, while for scientists, visualization processes developed in conjunction with understanding of function. Current findings further support Blazhenkova \& Kozhevnikov (2016) conclusions that visualization plays a different role and it is used for different purposes in teams of different specializations. In particular, visual artists tend to use their visual imagery as a source of creative inspiration for generation of visual aesthetic representations, while scientists tend to use imagery for solving problems and communication of unambiguous meanings that have functional relevance (see also Gooding, 2004; Rosenberg \& Trusheim, 1989). In contrast to scientists, both visual artists and mixed teams were more concerned with visual appearance than with functional characteristics of a creative work, which is possibly happened, because the task was interpreted as more artistic than scientific challenge. Furthermore, the relatively high proportion of organizational processes in mixed groups is likely to reflect the challenges that they had to deal with due to their diversity. Indeed, literature suggested that diversity, even while being potentially beneficial to team performance, may cause coordination difficulties (Cronin \& Weingart, 2007; Milliken, Bartel, \& Kurtzberg, 2003). As was evident in our study, coordination difficulties may have resulted in the increased number of organizational collaborative processes that involved votes and regulations. Moreover, the case of mixed teams demonstrated that the presence of members who are competent in a certain domain of knowledge (e.g., visual art) 
may be beneficial for all other members in the group, and may lead to success in at least some aspects of team performance (e.g., increasing artistic quality of the collaborative product). Indeed, there is growing evidence that both, artists and scientists, can benefit from their collaboration (Edmonds \& Leggett, 2010; Keefe, Karelitz, Vote, \& Laidlaw, 2005; Meyer, Staples, Minneman, Naimark, \& Glassner, 1998). For example, Keefe et al. described benefits of artistic collaboration in designing virtual reality scientific visualizations and claimed that artists can provide a unique source of visual insight in tackling difficult visual problems, as well as in analysing and refining visual works. However, the present research suggests that such collaboration may not equally benefit the team performance in all aspects. In particular, the presence of visual artists in the team may provide visual insights and considerably improve the artistic quality of the creative product; however, this can happen at the expense of scientific clarity of the creative output or at the expense of time devoted to coordination and organizational activities.

Compared to all other groups, humanities team had the lowest frequency of mental simulation visualization category in the protocol analysis. Besides, the majority of humanities team critical decisions (as reflected in critical moves) were unrelated to visualization processes. Moreover, humanities team did not use visualization for either predicting the contents of a creative drawing or for creating new knowledge. The protocol analysis of visualization categories and their dynamic distribution suggests that humanities team did not use visualization for planning and prediction, but rather for interpretation of already drawn content. Markedly, humanities team generated their main ideas only at the end of the drawing. Unlike humanities team, visual art, mixed, and science teams generated their key creative ideas at the very beginning of the creative process (as reflected by the entropy dynamics). In addition, the examination of time spent for planning activities showed that humanities team did not spend much time for planning prior to drawing and did not use visualization for planning, while other teams, before the start of their drawing, devoted a significant portion of time for planning that involved visualizing and drafting of a future work. These results are consistent with other research that compared experts versus novices' performance and reported that expert scientists and designers employ earlier planning and prediction during the problem solving as compared to non-experts. For example, Atman et al. (1999) found that expert engineering students, compared to novices, paid more attention to 'problem scoping' or adequately setting up the problem before beginning the analysis. Similarly, in the field of design, Christiaans and Dorst (1992) compared junior and senior industrial design students and found that that senior students tended to clarify priorities early on in the process. In the present study, humanities team could be considered as non-expects, since their skills may be incongruent to the current creative task requirements, involving more visual rather than verbal processing.

Furthermore, the present study supported the link between the team processes 
and performance. Linkography metrics of different specialization teams appeared to be partially consistent with professionals' evaluations of these drawings reported in Blazhenkova and Kozhevnikov (2016). This previous study showed that visual art and mixed teams' drawings were estimated as the highest in artistic quality, science teams were estimated as the highest in concept clarity, whereas humanities drawings were evaluated as the lowest on both criteria. The present results suggest that common Linkography measures, Link Index and Entropy, can predict professionals' estimates of creative outputs only in specific aspects of productivity: artistic quality, but not conceptual clarity. Thus, Linkograph link interconnectedness and completeness measures may not be relevant for a prediction of all aspects of the idea development and creativity. Overall, the present research shows the possibilities of Linkography method for understanding cognitive processes underlying group creative performance. At the same time, the present findings have potential applications for the field of design. The structure of a Linkograph (dynamics of its density, location of critical moves) can provide useful information about the group dynamic processes and employed strategies. The possibility to detect and quantify the strategical characteristics of a team processes can be very critical for predicting team performance. This would considerably contribute to the existing research examining the characteristics of team creative processes and aid understanding how the implementation of successful strategies contributes group performance (Cronin \& Weingart, 2007; Hackman \& Hackman, 2002; Salas et al., 2005). Furthermore, our study provides the case of integrative examination of the relative frequency of protocol categories (parsing out different visualization and non-visual processes), their dynamics throughout the creative progress, and Linkograph metrics (e.g., critical moves) considered in relation to the identified protocol categories. Such an approach may inspire the development of new methods and measures in the field of design research for assessing different aspects of creative productivity (e.g., not only artistic creativity, but also scientific creativity related to functional characteristics) as well as for assessing cognitive processes (e.g., different aspects of visualization). Our research shows the possibilities of Linkography methodology for understanding cognitive aspects of creative team processes and suggests new perspectives in implementing Linkography measures.

One of the major limitations of the current study is implementing the case approach and using adolescents, which put restrictions on the possible generalizations for adult population. Nevertheless, the present study brings new knowledge about the role of visualization for the creative processes in teams of different specializations. Our research sheds light on understanding creative visual processes and dynamics behind multidisciplinary creative team interaction, and in particular, how visual information is processed and shared among the members of the teams with different visualization profiles. Our research emphasizes the importance of considering differences in visualization profiles while com- 
posing teams of different specializations. Here we show the case that such differences have a considerable impact on team process characteristics, which in turn are related with the quality characteristics of the final creative outputs. In response to growing interest in art-science interdisciplinary collaboration, current research brings new insights about the possibilities and limitations of creative collaboration between individuals with different specializations. Present findings should be interesting for a broad audience from cognitive, educational and organizational psychology as well as design research and other applied fields.

\section{Acknowledgements}

This work was partially supported by National Institute of Education (Singapore) under the MOE Academies of Fund, AFR 0117 MK to Maria Kozhevnikov.

\section{Conflicts of Interest}

The authors declare no conflicts of interest regarding the publication of this paper.

\section{References}

Aggarwal, I., \& Woolley, A. W. (2013). Do You See What I See? The Effect of Members' Cognitive Styles on Team Processes and Errors in Task Execution. Organizational Behavior and Human Decision Processes, 122, 92-99.

https://doi.org/10.1016/j.obhdp.2013.04.003

Atman, C. J., Chimka, J. R., Bursic, K. M., \& Nachtmann, H. (1999). A Comparison of Freshman and Senior Engineering Design Processes. Design Studies, 20, 131-152. https://doi.org/10.1016/S0142-694X(98)00031-3

Austin, S., Steele, J., Macmillan, S., Kirby, P., \& Spence, R. (2001). Mapping the Conceptual Design Activity of Interdisciplinary Teams. Design Studies, 22, 211-232. https://doi.org/10.1016/S0142-694X(00)00026-0

Banks, A. P., \& Millward, L. J. (2000). Running Shared Mental Models as a Distributed Cognitive Process. British Journal of Psychology, 91, 513-531. https://doi.org/10.1348/000712600161961

Bilda, Z., Costello, B., \& Amitani, S. (2006). Collaborative Analysis Framework for Evaluating Interactive Art Experience. CoDesign, 2, 225-238. https://doi.org/10.1080/15710880601008026

Blazhenkova, O., \& Kozhevnikov, M. (2010). Visual-Object Ability: A New Dimension of Non-Verbal Intelligence. Cognition, 117, 276-301. https://doi.org/10.1016/j.cognition.2010.08.021

Blazhenkova, O., \& Kozhevnikov, M. (2016). Types of Creativity and Visualization in Teams of Different Educational Specialization. Creativity Research Journal, 28, 123-135. https://doi.org/10.1080/10400419.2016.1162638

Blazhenkova, O., Kozhevnikov, M., \& Becker, M. (2011). Object-Spatial Imagery and Cognitive Styles in Children and Adolescents: Developmental Trajectories in Relation to Ability. Learning and Individual Differences, 21, 281-287. https://doi.org/10.1016/j.lindif.2010.11.012

Cai, H., Do, E. Y.-L., \& Zimring, C. M. (2010). Extended Linkography and Distance 
Graph in Design Evaluation: An Empirical Study of the Dual Effects of Inspiration Sources in Creative Design. Design Studies, 31, 146-168.

https://doi.org/10.1016/j.destud.2009.12.003

Cannon-Bowers, J. A., Salas, E., \& Converse, S. (1993). Shared Mental Models in Expert Team Decision Making. In N. J. Castellan Jr. (Ed.), Individual and Group Decision Making: Current Issues (pp. 221-246). Hillsdale, NJ: Lawrence Erlbaum.

Christiaans, H., \& Dorst, K. (1992). An Empirical Study into Design Thinking. In N. Cross, K. Dorst, \& N. Roozenburg (Eds.), Research in Design Thinking (pp. 119-126). Delft: Delft University Press.

Cronin, M. A., \& Weingart, L. R. (2007). Representational Gaps, Information Processing, and Conflict in Functionally Diverse Teams. Academy of Management Review, 32, 761-774. https://doi.org/10.5465/amr.2007.25275511

Dong, A. (2005). The Latent Semantic Approach to Studying Design Team Communication. Design Studies, 26, 445-461. https://doi.org/10.1016/j.destud.2004.10.003

Dunbar, K. (1999). The Scientist in Vivo: How Scientists Think and Reason in the Laboratory. In L. Magnani, N. Nersessian, \& P. Thagard (Eds). Model-Based Reasoning in Scientific Discovery (pp. 89-98). New York: Plenum Press.

Edmonds, E., \& Leggett, M. (2010). How Artists Fit into the Research Processes. Leonardo, 43, 194-195. https://doi.org/10.1162/leon.2010.43.2.194

Egan, T. M. (2005). Creativity in the Context of Team Diversity: Team Leader Perspectives. Advances in Developing Human Resources, 7, 207-225. https://doi.org/10.1177/1523422305274526

Goldschmidt, G. (1990). Linkography: Assessing Design Productivity. In R. Trappl (Ed.), Cybernetics and Systems 90. Proceedings of the Tenth European Meeting on Cybernetics and Systems Research (pp. 291-298). Singapore: World Scientific.

Goldschmidt, G. (1992). Criteria for Design Evaluation: A Process-Oriented Paradigm. In Y. E. Kalay (Ed.), Evaluating and Predicting Design (pp. 67-79). New York: Wiley. https://doi.org/10.1016/0142-694X(94)00009-3

Goldschmidt, G. (1995). The Designer as a Team of One. Design Studies, 16, 189-209.

Goldschmidt, G., \& Tatsa, D. (2005). How Good Are Good Ideas? Correlates of Design Creativity. Design Studies, 26, 593-611. https://doi.org/10.1016/j.destud.2005.02.004

Gooding, D. (2004). Cognition, Construction and Culture: Visual Theories in the Sciences. Journal of Culture and Cognition, 4, 551-593. https://doi.org/10.1163/1568537042484896

Hackman, J. R., \& Hackman, R. J. (2002). Leading Teams: Setting the Stage for Great Performances. Brighton, MA: Harvard Business Press.

Hagaman, S. (1990). The Community of Inquiry: An Approach to Collaborative Learning. Studies in Art Education, 31, 149-157. https://doi.org/10.2307/1320762

Hirsch, P. L., Shwom, B. L., Yarnoff, C., Anderson, J. C., Kelso, D. M., Olson, G. B., \& Colgate, J. E. (2001). Engineering Design and Communication: The Case for Interdisciplinary Collaboration. International Journal of Engineering Education, 17, 343-348.

Kan, J. W. T., Bilda, Z., \& Gero, J. S. (2007). Comparing Entropy Measures of Idea Links in Design Protocols: Linkography Entropy Measurement and Analysis of Differently Conditioned Design Sessions. Artificial Intelligence for Engineering Design, Analysis and Manufacturing, 21, 367-377. https://doi.org/10.1017/S0890060407000339

Kan, J. W., \& Gero, J. S. (2005). Can Entropy Indicate the Richness of Idea Generation in Team Designing. In Proceedings of the 10th International Conference on Computer 
Aided Architectural Design Research in Asia (pp. 451-457). New Delhi, India: TVB.

Kan, J. W. T., \& Gero, J. S. (2007). Can an Objective Measurement of Design Protocols Reflect the Quality of a Design Outcome? In ICED07 (pp. 1-12). Paris: Ecole Centrale de Paris.

Kan, J. W. T., \& Gero, J. S. (2008). Acquiring Information from Linkography in Protocol Studies of Designers. Design Studies, 29, 315-337. https://doi.org/10.1016/j.destud.2008.03.001

Kan, J. W. T., \& Gero, J. S. (2009). Using the FBS Ontology to Capture Semantic Design Information in Design Protocol Studies. In J. McDonnell, \& P. Lloyd (Eds.), About: Designing. Analysing Design Meetings (pp. 213-229). Boca Raton, FL: CRC Press.

Keefe, D., Karelitz, D., Vote, E., \& Laidlaw, D. H. (2005). Artistic Collaboration in Designing VR Visualizations. IEEE Computer Graphics and Applications, 25, 18-23. https://doi.org/10.1109/MCG.2005.34

Klimoski, R., \& Mohammed, S. (1994). Team Mental Model: Construct or Metaphor? Journal of Management, 20, 403-437. https://doi.org/10.1177/014920639402000206

Kozbelt, A. (2001). Artists as Experts in Visual Cognition. Visual Cognition, 8, 705-723. https://doi.org/10.1080/13506280042000090

Kozhevnikov, M., Blazhenkova, O., \& Becker, M. (2010). Trade-Off in Object versus Spatial Visualization Abilities: Restriction in the Development of Visual Processing Resources. Psychonomic Bulletin \& Review, 17, 29-35.

https://doi.org/10.3758/PBR.17.1.29

Kozhevnikov, M., Kozhevnikov, M., Chen, J. Y., \& Blazhenkova, O. (2013). Creativity, Visualization Abilities, and Visual Cognitive Style. British Journal of Educational Psychology, 83, 196-209. https://doi.org/10.1111/bjep.12013

Kozhevnikov, M., Kosslyn, S. M., \& Shepard, J. (2005). Spatial versus Object Visualizers: A New Characterization of Visual Cognitive Style. Memory and Cognition, 33, 710-726. https://doi.org/10.3758/BF03195337

Kraiger, K., \& Wenzel, L. H. (1997). Conceptual Development and Empirical Evaluation of Measures of Shared Mental Models as Indicators of Team Effectiveness. In M. T. Brannick, E. Salas, \& E. Prince (Eds.), Team Performance Assessment and Measurement (pp. 63-84). Mahwah, NJ: Lawrence Erlbaum Associates.

Kvan, T. (2000). Collaborative Design: What Is It? Automation in Construction, 9, 409-415. https://doi.org/10.1016/S0926-5805(99)00025-4

Mesmer-Magnus, J. R., \& DeChurch, L. A. (2009). Information Sharing and Team Performance: A Meta-Analysis. Journal of Applied Psychology, 94, 535-546. https://doi.org/10.1037/a0013773

Meyer, J., Staples, L., Minneman, S., Naimark, M., \& Glassner, A. (1998). Artists and Technologists Working Together (Panel). In Proceedings of the 11th Annual ACM Symposium on User Interface Software and Technology (pp. 67-69). New York: ACM Press. https://doi.org/10.1145/288392.289101

Miller, A. I. (1996). Insights of Genius Imagery and Creativity in Science and Art. Cambridge, MA: MIT Press.

Milliken, F. J., Bartel, C., \& Kurtzberg, T. (2003). Diversity and Creativity in Work Groups: A Dynamic Perspective on the Affective and Cognitive Processes That Link Diversity and Performance. In P. B. Paulus, \& B. Nijstad (Eds.), Group Creativity (pp. 32-62). Oxford: Oxford University Press. https://doi.org/10.1093/acprof:oso/9780195147308.003.0003

Pourmohamadi, M., \& Gero, J. S. (2011). Linkographer: An Analysis Tool to Study De- 
sign Protocols Based on FBS Coding Scheme. In S. Culley, B. Hicks, T. McAloone, T. Howard, \& Y. Reich (Eds.), Design Theory and Methodology (pp. 294-303). Glasgow: Design Society.

Rosenberg, H. S., \& Trusheim, W. (1989). Creative Transformations: How Visual Artists, Musicians, and Dancers Use Mental Imagery in Their Work. In P. Robin, \& J. E. Schorr (Eds.), Imagery: Current Perspectives (pp. 55-75). New York: Plenum Press. https://doi.org/10.1007/978-1-4899-0876-6_6

Salas, E., Sims, D. E., \& Burke, C. S. (2005). Is There a "Big Five" in Teamwork?. Small Group Research, 36, 555-599. https://doi.org/10.1177/1046496405277134

Shannon, C. E. (1948). A Mathematical Theory of Communication. The Bell System Technical Journal, 27, 397-423. https://doi.org/10.1002/j.1538-7305.1948.tb01338.x

Sonnenwald, D. H. (1996). Communication Roles That Support Collaboration during the Design Process. Design Studies, 17, 277-301. https://doi.org/10.1016/0142-694X(96)00002-6

Stevelt-Kaser, K., Pennington-Busick, S., \& Rhoades, M. (2004). Redefining the Principles and Elements of Educating Artists in the 21st Century. Columbus, OH: Ohio State University.

Stokols, D., Hall, K. L., Taylor, B. K., \& Moser, R. P. (2008). The Science of Team Science: Overview of the Field and Introduction to the Supplement. American Journal of Preventive Medicine, 35, S77-S89. https://doi.org/10.1016/j.amepre.2008.05.002

Terenzini, P. T., Cabrera, A. F., Colbeck, C. L., Parente, J. M., \& Bjorklund, S. A. (2001). Collaborative Learning vs. Lecture/Discussion: Students' Reported Learning Gains. Journal of Engineering Education, 90, 123-130. https://doi.org/10.1002/j.2168-9830.2001.tb00579.x

Van der Lugt, R. (2003). Relating the Quality of the Idea Generation Process to the Quality of the Resulting Design Ideas. In DS 31: Proceedings of ICED 03, the 14th International Conference on Engineering Design (pp. 601-602). Glasgow, UK: Design Society.

Woolley, A. W., Chabris, C. F., Pentland, A., Hashmi, N., \& Malone, T. W. (2010). Evidence for a Collective Intelligence Factor in the Performance of Human Groups. Science, 330, 686-688. https://doi.org/10.1126/science.1193147

Woolley, A. W., Hackman, J. R., Jerde, T. E., Chabris, C. F., Bennett, S. L., \& Kosslyn, S.M. (2007). Using Brain-Based Measures to Compose Teams: How Individual Capabilities and Team Collaboration Strategies Jointly Shape Performance. Social Neuroscience, 2, 96-105. https://doi.org/10.1080/17470910701363041

Wuchty, S., Jones, B. F., \& Uzzi, B. (2007). The Increasing Dominance of Teams in Production of Knowledge. Science, 316, 1036-1039.

https://doi.org/10.1126/science.1136099 


\section{Appendices}

\section{A. AN EXAMPLE OF A LINKOGRAPH}

Figure A1 provides an example of linking in a fragment of the Linkograph, where the segments identified in the protocol analyses are represented as a sequence, and the links between meaningfully associated segments are drawn. Segments 16 and 17 are linked to segment 8, because they all express the idea of cubical/squared shape of the planet, and additionally, segments 16 and 17 are connected to each other because they both present the idea of rings surrounding the planet. A node represented as a filled circle indicates a relation between any two segments, and it appears at the intersection of the links. Segment 17 is backlinked to segments 16 and 8 , and segment 8 is forelinked to segments 16 and 17 .

\begin{tabular}{|c|c|c|}
\hline Segments & Idea Links & Content \\
\hline Segment 8 & & $\begin{array}{l}\text { "Let's make the planet shaped as } \\
\text { a square" }\end{array}$ \\
\hline$\cdots$ & & $\ldots$ \\
\hline Segment 16 & & $\begin{array}{l}\text { "Let's draw it like a cube with the } \\
\text { rings around, as in Jupiter" }\end{array}$ \\
\hline Segment 17 & & $\begin{array}{l}\text { "No, if it is square-shaped, then } \\
\text { there can't be rings" }\end{array}$ \\
\hline
\end{tabular}

Figure A1. Example of a Linkograph fragment.

\section{B. EXAMPLES OF LINKOGRAPH STRUCTURES}

Figure A2 provides examples of some possible Linkograph structures and their interpretations.

\begin{tabular}{|c|c|}
\hline$\bullet \bullet \bullet$ & $\begin{array}{l}\text { Five segments are totally unrelated, indicating no } \\
\text { connection between ideas, hence low opportunity for } \\
\text { idea development. }\end{array}$ \\
\hline & $\begin{array}{l}\text { All segments are interconnected; this shows that this is a } \\
\text { totally integrated process with no diversification, } \\
\text { suggesting that a premature crystallization or fixation on } \\
\text { one idea may have occurred, therefore there is a very low } \\
\text { opportunity for novel ideas. }\end{array}$ \\
\hline & $\begin{array}{l}\text { Segments are related only to directly preceding } \\
\text { segments. This indicates the process is progressing but } \\
\text { not developing, indicating some opportunities for idea } \\
\text { development. }\end{array}$ \\
\hline & $\begin{array}{l}\text { Moves are interrelated but not totally connected, } \\
\text { indicating that there are many opportunities for idea } \\
\text { development. }\end{array}$ \\
\hline
\end{tabular}

Figure A2. Examples of some possible linkograph structures and their interpretations. Note. This Figure was modified from Kan and Gero, 2008. 